\title{
Respuestas del Trabajo Social ante emergencias sociales y problemáticas sociales complejas de México y España
}

\author{
Breogán Riobóo-Lois \\ Trabajador social \\ Integra-T Consultoría Social. Ourense, España \\ https://orcid.org/0000-0002-7293-1694 • arioboo@uvigo.es
}

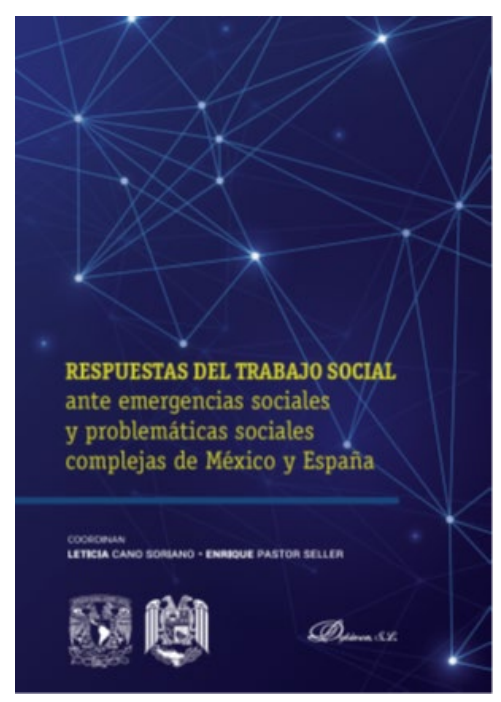
Cano-Soriano, L. y Pastor-Seller, E. (Coords.) 2019.

Respuestas del Trabajo Social ante emergencias sociales y problemáticas sociales complejas de México y España.

Madrid, España: Dykinson.

303 p.

ISBN: 978-84-1324-702-1

Respuestas del Trabajo Social dibuja, mediante diferentes investigadores y especialistas del Trabajo Social, el contexto en el que transita la intervención en emergencias sociales y la falta de trasvase que, desde la disciplina, está habiendo en el diseño de políticas públicas para la prevención y la protección de la población, especialmente aquella más vulnerable.

Nos encontramos ante una obra colectiva, encabezada por los docentes e investigadores de Trabajo Social Leticia Cano Soriano y Enrique Pastor Seller. El libro contiene una valiosa compilación de catorce trabajos inéditos, siete de los cuales provienen Recibido: 03/07/2020 | Aprobado: 07/10/2020 | Publicado: 01/01/2021

\section{¿Cómo citar este artículo? / How to quote this article?}

Riobóo-Lois, B. (2021). Respuestas del Trabajo Social ante emergencias sociales y problemáticas sociales complejas de México y España. Prospectiva. Revista de Trabajo Social e intervención social, (31), 463-467. doi: 10.25100/prts.v0i31.10124. 
Riobóo-Lois

de México y otros siete de España, dotándose así de un enriquecedor análisis transoceánico. Este hecho permite una lectura de carácter internacional y de total actualidad, atendiendo al fenómeno del confinamiento mundial reciente, debido al covid-19.

Por otra parte, la obra cuenta con autoras y autores de prestigio de universidades mexicanas (Autónoma de México y Autónoma Metropolitana) y españolas (Murcia, Jaén, Vigo, Salamanca, Valencia, Almería, Málaga, y UNED). Asimismo, cuenta con aportaciones de profesionales externos al ámbito docente de entidades de ambos países como Sensodanza Terapia A.C. en México y el Instituto Foral Acción Social de la Diputación Foral de Vizcaya. A partir de la presentación de dos conceptos similares a la vez que divergentes, se traza el hilo conductor de las diferentes obras con las que se configura este libro. De un lado, las catástrofes causadas por fenómenos naturales y del otro, las calamidades tras las que aparece la acción u omisión del ser humano.

El primer capítulo presenta el fenómeno del desplazamiento forzado interno (DFI) como elemento clave de la crisis humanitaria, identificando tres causas en México: violencia de organizaciones delictivas y del Estado, megaproyectos que provocan violencia contra las comunidades afectadas y desastres naturales que se utilizan para invisibilizar las causas estructurales que sustentan dichas acciones violentas. Consecuentemente, se generan dos clases de poblaciones afectadas: la que padece los efectos directos (primaria) y aquellas que los sufren de forma indirecta en el territorio (secundaria) o sin habitar en éste (terciaria). Los autores determinan, con buen criterio, que todo desastre es social pese a no ser asumidos así, reduciendo sus efectos a lo inmediato y derivando sus esfuerzos a la atención de la emergencia. A continuación, identifican tres elementos que impiden el éxito de las intervenciones postdesastre: la imposición de proyectos de reubicación y reconstrucción limitados a la vivienda, la reubicación en zonas de igual o mayor riesgo y las reubicaciones que buscan despojar a las comunidades de sus espacios de vida y trabajo. Concluyen la existencia de una interrelación entre daños y pérdidas y las decisiones políticas, por lo que sólo mediante proyectos de reconstrucción en base a los derechos humanos y la participación de las personas desplazadas podrá diseñarse una gestión integral de riesgos para su prevención y mitigación a futuro.

Mediante el segundo capítulo, a partir de una sistemática conceptualización de las diferencias existentes entre una emergencia y una catástrofe, la autora nos presenta un modelo de intervención en emergencias basado en el enfoque temporal. Este planteamiento significa concebir la intervención en emergencias como un proceso a lo largo del tiempo y no como una acción puntual y localizada. A través de la clasificación de las fases o etapas de la emergencia afirma que el conocimiento del momento en el que se encuentra la población objetivo es clave para una intervención eficaz y eficiente. Seguidamente formula los objetivos y estrategias de intervención del Trabajo Social estructurando ampliamente las 
funciones de la profesión en base a las necesidades específicas que subyacen según las etapas de: preemergencia, emergencia y postemergencia.

Discapacidad y emergencia social por fenómenos naturales: reflexiones sobre el 19S, es el título que recibe el tercer capítulo que parte del sismo que tuvo lugar el 19 de septiembre del 2017 en el golfo de Tehuantepec, México. Ante las actuaciones llevadas a cabo durante esa emergencia, las autoras consideran la "necesidad de trabajar de manera colectiva las acciones a seguir (...) con diversos grupos poblacionales" (Lázaro, Cruz, Pérez y Hernández, 2019, p. 79), en especial las personas mayores y/o con discapacidad, dotando de mayor integralidad a la intervención. Finaliza el capítulo, analizando la aportación del Trabajo Social al ámbito de la protección civil en dos ejes: la disminución de riesgos desde la comunidad y la emergencia social.

El cuarto capítulo se centra en "la epidemia de la soledad" (De la Fuente, Martín y Ortega, 2019, p. 94). A partir de una comparativa internacional especialmente centrada en Europa y América Latina, se procede a analizar los protocolos de actuación y coordinación entre los servicios sociales comunitarios y las unidades de Trabajo Social hospitalario en España. Seguidamente nos presentan diferentes alternativas para luchar frente a soledad, concluyendo en la necesidad de soluciones innovadoras y especializadas que respeten y mantengan derechos fundamentales con capacidad de adaptarse a nuevos desafíos.

Julia del Carmen Chávez Carapia analiza el fenómeno de La violencia familiar antesala de los feminicidios en el quinto capítulo. A través del análisis de la situación de la violencia de género en México, la autora nos expone la pervivencia de la cultura patriarcal cuyo máximo exponente acaban siendo una cantidad importante de feminicidios. Finaliza el capítulo destacando la importancia del Trabajo Social mediante métodos socioeducativos que incluyan la perspectiva de género buscando la mejora de las condiciones de vida y la transformación social.

En el sexto capítulo, Carmen Verde-Diego, Antonia Picornell-Lucas y José Javier Navarro-Pérez nos adentran en clave analítica en el sistema de protección a la infancia y la adolescencia en España. Clarifican sus principios y los conceptos de situación de riesgo y de desamparo antes de presentar de forma resumida los datos estadísticos de las medidas de protección contempladas. La introducción de estos conceptos y datos de relevancia da pie al análisis de las fortalezas y debilidades del sistema de protección a la infancia, para finalizar el capítulo proponiendo la evaluación de los riesgos como medida de intervención social ante la desprotección infantil.

La violencia estructural en México se aborda en los capítulos séptimo y duodécimo. En el séptimo, se relacionan pobreza, violencia estructural y clientelismo político para exponer después el enfoque desde el Trabajo Social antes de finalizar con una serie de 
Riobóo-Lois

recomendaciones. Mientras, en el duodécimo, se analiza la relación entre la primera infancia y las violencias estructurales para abordar también la intervención desde la disciplina en estos contextos.

El capítulo octavo se centra en la precariedad económica en España, analizando la distribución competencial en los distintos niveles administrativos, así como las prestaciones locales, la respuesta desde otras entidades o la sociedad civil y las provenientes del Trabajo Social.

Joaquín Castillo de Mesa y Antonio López Peláez nos presentan un capítulo noveno realmente novedoso. En él analizan el uso de las redes sociales, especialmente Twitter, en situaciones de emergencia social, proponiendo diferentes ejemplos e identificando diferentes colectivos que se constituyen como interlocutores influyentes en estas situaciones, difundiendo distintos tipos de información relevante. Finalmente, identifican tres áreas importantes desde la perspectiva del Trabajo Social en las redes sociales: la formación, la institucionalización del trabajo social virtual y las estrategias de intervención. El décimo capítulo se trata de un estudio estadístico descriptivo sobre La concentración espacial de la práctica de las cesáreas en México entre los años 2011 y 2014. Las autoras entienden que hay cabida para la acción desde el Trabajo Social, por ejemplo, con mujeres gestantes y el desconocimiento o desinformación que puedan tener acerca de los riesgos de las cesáreas.

De la mano de Rubén González-Rodríguez y Enrique Pastor-Seller, nos adentramos en el Sistema para la Autonomía y Atención a la Dependencia (SAAD) en el undécimo capítulo. Tras detallarnos sus aspectos más importantes, analizan las situaciones de urgencia y emergencia social que se abordan dentro del SAAD para identificar, después, cuál es la respuesta a estas situaciones desde el Trabajo Social. Certeramente, los autores anuncian la necesidad de concretar "en mayor medida las situaciones que deben ser atendidas como emergencia", además de "la existencia de indicadores que permitan un marco en el que poder objetivar que ninguna situación personal que necesita atención quede sin atender" (González-Rodríguez y Pastor-Seller, 2019, p. 238).

El décimo tercer capítulo nos sume en un tema fascinante, el de las migraciones, en este caso de México a los Estados Unidos de Norteamérica. Mediante entrevistas semiestructuradas, los autores nos presentan un estudio cualitativo basado en la memoria oral de las propias protagonistas. Desde que surge la idea o necesidad, pasando por el cómo afrontar el viaje y la llegada final a su destino, donde se manifiestan una serie de consecuencias que identifican concisamente, en tres fases: aculturación, necesidad de volver a su ciudad de origen y desestructuración familiar.

Finaliza el libro con un último capítulo que aborda el caso de Ayotzinapa, en México, donde 43 estudiantes desaparecieron el 26 de septiembre de 2014. A través del análisis de la 
Riobóo-Lois

actuación gubernamental según su concepto de seguridad y de los discursos respecto a la desaparición de estas personas se trazan sus representaciones sociales. Se expone la aquiescencia de la disciplina respecto a los intereses del Estado según su evolución reciente, para finalmente analizar dos modelos contrapuestos de acción ante estas situaciones desde el Trabajo Social: un dispositivo de intervención o un dispositivo de intercesión.

Por lo tanto, estamos ante una obra de gran interés para la profesión y de especial relevancia en los tiempos actuales, en los que las situaciones de emergencia social parecen multiplicarse y extenderse de forma continua. Este libro nos aporta diferentes teorías, enfoques y métodos de intervención para el Trabajo Social, con una visión amplia e internacional. Con su lectura, no sólo refrescaremos conocimientos que se hayan ido aletargando en nuestra cabeza, sino que se nos abrirá todo un nuevo mapa de oportunidades y de saberes profesionales que nos serán de gran ayuda en nuestro día a día.

En resumen, se trata de una obra altamente recomendable, tanto para profesionales del Trabajo Social, como incluso de otras disciplinas que tengan interés por la intervención en situaciones de catástrofe o calamidad. Además, es una obra magnífica para la lectura por parte de futuras profesionales del Trabajo Social, estudiantes que tengan interés por descubrir diferentes ámbitos de actuación de la profesión que puedan llegar a abrirle los ojos de cara a su futura dedicación profesional.

\section{Referencias bibliográficas}

De la Fuente, Y. M., Martín, M. C., y Ortega, T. (2019). El rostro de la desolación. La soledad no elegida en el envejecimiento. En L. Cano-Soriano y E. Pastor-Seller (Coords.), Respuestas del Trabajo Social ante emergencias sociales y problemáticas sociales complejas de México y España (pp. 93-113). Madrid, España: Dykinson.

González-Rodríguez, R., y Pastor-Seller, E. (2019). Realidades de emergencia social en personas en situación de dependencia: indicadores y respuestas por parte del sistema de servicios sociales en España. En L. Cano-Soriano y E. Pastor-Seller (Coords.), Respuestas del Trabajo Social ante emergencias sociales y problemáticas sociales complejas de México y España (pp. 223-240). Madrid, España: Dykinson.

Lázaro, E., Cruz, N., Pérez, B., y Hernández, K. A. (2019). Discapacidad y emergencia social por fenómenos naturales: reflexiones sobre el 19S. En L. Cano-Soriano y E. PastorSeller (Coords.), Respuestas del Trabajo Social ante emergencias sociales y problemáticas sociales complejas de México y España (pp. 73-91). Madrid, España: Dykinson. 


\section{OTROS ARTÍCULOS DE PROSPECTIVA No. 31 DE 2021}

\section{PRESENTACIÓN}

Presentación. Reflexiones sobre desafios al publicar sistematizaciones

Rosa María Cifuentes-Gil

\section{EDITORIAL}

Reflexiones sobre Trabajo Social: aportes de la Sistematización

María Rocío Cifuentes-Patiño

\section{ARTÍCULOS}

Hacer lo que se sabe, pensar lo que se hace. La sistematización como modalidad investigativa Alfonso Torres-Carrillo

Aportes y desafios de la Sistematización de experiencias en el Trabajo Social y la extensión crítica. Apuntes y reflexiones desde la perspectiva de la Educación Popular

María Rosa Goldar

Valeria Chiavetta

La sistematización en Trabajo Social y la epistemología feminista del punto de vista. Diálogos sobre la producción de conocimiento sustentada en experiencias

Ruth Noemí Parola

María Florencia Linardelli

La Sistematización investigativa de las experiencias: del baile de los que sobran a la fiesta de los que faltan

María Belén Ortega-Senet

Sistematización y Trabajo Social en Chile. El largo y sinuoso camino

Patricia Lorena Castañeda-Meneses

Ana María Salamé-Coulon
Sentipensar la pandemia COVID-19 desde la sistematización de la experiencia en Trabajo Social: reflexiones del profesor Oscar Jara Holliday

Elia Sepúlveda-Hernández

La sistematización de experiencias, una investigación social cualitativa que potencia buenas prácticas de convivencia y gobierno. La experiencia de un conjunto residencial multifamiliar en Cali, Colombia Martha Lucia Echeverry-Velásquez Manuela Prada-Dávila

Construcción de subjetividades epistemológicaspolíticas de profesoras y profesores de Investigación social en una universidad privada y confesional en Bogotá

Giovanni Mora-Lemus

Sistematización de la experiencia Reconocimiento de los derechos humanos del adulto mayor en dos familias residentes en Cali y Valledupar (Colombia)

Lina María Cuello-Lacouture

Jimena del Pilar Jaramillo-Jaramillo

La memoria transformadora como estrategia de intervención profesional en los procesos de reconciliación social: comprensión a partir de mujeres campesinas, excombatientes y jóvenes en Manizales, Colombia

Yeimmy Stephania Corredor-Sotelo

Juliana Fuertes-Fuertes

Sistematización de una estrategia de educación informal implementada en personas privadas de la libertad en el establecimiento penitenciario de mediana seguridad y carcelario de Barranquilla, Colombia

Rafael Humberto Herrera-Mercado Rafael Alberto Zambrano-Vanegas 
Aportes significativos del proceso de intervención comunitaria con la Escuela Popular de Comunicación Alternativa Jaime Garzón de la ciudad de Cúcuta, Colombia

Carlos Lasso-Urbano

La sistematización de la intervención como metodología de investigación en Trabajo Social. Importancia práctica y teórica de la fase de recogida de datos en la intervención social según experiencia del Programa de Apoyo a las Familias en Zaragoza, España

Elisa Esteban-Carbonell

Nuria Del Olmo-Vicén

Papel de la sistematización de experiencias en los procesos de evaluación de intervenciones de salud pública en la Comuna Saludable por la Paz, Cali - Colombia

Jenny Faisury Peña-Varón

Paola Andrea Marín-Velásquez

Janeth Mosquera-Becerra

Experiencia de intervención social en hogares comunitarios integrales del barrio Alfonso Bonilla Aragón, Cali - Colombia

Julián Alexander Montaño-Cárdenas

Las políticas sociales y el gobierno de la "población indígena". Estrategias y regulaciones en el multiculturalismo chileno

Rodrigo Agustín Navarrete-Saavedra

Representaciones sociales sobre estilo de autoridad y tipos de interacción en cuidadores de residencias de protección infantil en Chile

Marcelo Gallegos-Fuentes
Carmen Gloria Jarpa-Arriagada

Reflexiones sobre inseguridad social y cuestiones penales. Una respuesta estratégica a partir de experiencias de cooperativismo con ex detenidos en Argentina

Analia Elizabeth Otero

Yael Yanina Barrera

Desarrollo y salud: la emergencia de un nuevo paradigma

Jesús María Sánchez-Ordóñez

Trabajo Social en ejercicio libre: la perspectiva profesional en España

Paula Frieiro-Padín

Tamara Fernández-Arias

Rubén González-Rodríguez

\section{RESEÑAS}

Social Work and the City: Urban Themes in 21stCentury Social Work

Felipe Saravia-Cortés

Respuestas del Trabajo Social ante emergencias sociales y problemáticas sociales complejas de México y España

Felipe Saravia-Cortés

El feminismo, el género y la profesionalización del trabajo social en Colombia (1936-2004)

Ambar Oriana Serna-Lombo

El puño invisible. Arte, revolución y un siglo de cambios culturales

Carlos Arturo Robledo-Marín

\section{PROSPECTIVA}

No. $31 \bullet$ ene.-jun. 2021

e-ISSN: 2389-993X • Universidad del Valle 